TITLE:

\title{
Methods for Designing Systems with Benefits of Inconvenience
}

$\operatorname{AUTHOR}(S)$ :

Kawakami, Hiroshi; Hiraoka, Toshihiro

CITATION:

Kawakami, Hiroshi ... [et al]. Methods for Designing Systems with Benefits of Inconvenience. Design, User Experience, and Usability. Design Philosophy and Theory 2019, 11583: 253-263

ISSUE DATE:

2019

URL:

http://hdl.handle.net/2433/252473

RIGHT:

This is a post-peer-review, pre-copyedit version of an article published in Design, User Experience, and Usability. Design Philosophy and Theory. The final authenticated version is available online at: https://doi.org/10.1007/978-3-030-235703_19.; この論文は出版社版でありません。引用の際には出版社版をご確認ご利用ください。; This is not the published version. Please cite only the published version. 


\title{
Methods for Designing Systems with Benefits of Inconvenience
}

\author{
H. Kawakami ${ }^{1}$ and T. Hiraoka ${ }^{2}$ \\ ${ }^{1}$ Kyoto University, Yoshida-honmachi, Sakyo, Kyoto, 606-8501 Japan \\ 2 Nagoya University, Chukusa, Nagoya, 4648601 Japan
}

\begin{abstract}
This paper introduces the concept of "benefits of inconvenience (BI)" and proposes ideation methods for designing humanmachine interaction (HMI) involving BI. HMI is the superset of HCI. Based on the assumption that convenience means saving time and reducing effort, efficiency, and high functionality provide convenience to users. On the other hand, for discussing HCI, interaction is essential that requires humans' time and efforts. In other words, inconvenience in the sense of above-mentioned assumption is essential for human-machine interactions. In this case, machines do not substitute humans but interact with humans, and inconvenience will be beneficial. This paper categorizes methods for designing systems with benefits of inconvenience into three classes.
\end{abstract}

Keywords: Benefits of Inconvenience, System Design, Human Machine Interaction

\section{Introduction}

\section{1 aaa}

Fuben-eki is Japanese that stands for further benefits of a kind of inconvenience [1]. It does not reflect nostalgia but provides a standpoint of reviewing existing things and designing new systems.

In general, designers tend to pursue designing convenient systems. Convenience may enrich our lives in some ways, but it is not always beneficial for users. Convenient systems may inflate such harms as excluding users, depriving the pleasure of using systems, and eroding human motivation and skills.

Authors of this paper conducted several workshops and practices for designing systems that provide users with benefits of inconvenience. Such practices revealed that ideation (conceiving ideas) processes of designers can be classified into three patterns as reported in Section 3 of this paper.

\section{Benefits of Inconvenience}

\subsection{Definition of Inconvenience}

Longman dictionary online defined the concept of convenience as "the quality of being suitable or useful for a particular purpose, especially by making something 
easier or saving you time," and inconvenience as "problems caused by something which annoy or affect you." This paper employs more simple definitions of convenience/inconvenience as follows:

convenience: saving labor to attain a specific task,

inconvenience: being comparatively not convenient, i.e., requiring more labor than convenience,

where the word labor has the following meanings:

physical labor: requiring time and effort,

mental labor: requiring special skills, including the consumption of such cognitive resources as paying attention, memorization, and conception.

Stemming from the fact that labor-derived benefits exist, those simple definitions allow us to think inconvenience does not contradict benefit.

\section{$2.2 \quad$ Fuben-eki Systems}

Systems that provide users with the benefits of inconvenience are called fubeneki systems. A conventional system design that produces convenient systems is evaluated in terms of one axis: the amount of labor. In this case, designers assume that convenience is always beneficial. In other words, as shown in Fig. 1, the axis of convenience/inconvenience and the axis of benefits/harms are identical or in the same direction.

$\stackrel{\text { Harms }}{\stackrel{\text { Benefits }}{\longrightarrow}}$

Fig. 1. Conventional understanding the relation between convenience and benefits

Fuben-eki system design, on the other hand, affirms that convenience is independent with benefits and the axis of convenience/inconvenience and the axis of benefits/harms are orthogonal as shown in Fig. 2. In this case, we get the quadrants. The upper right quadrant denotes benefits of convenience, the upper left quadrant denotes benefits of inconvenience, the lower left quadrant denotes harms of inconvenience, and the lower right quadrant denotes harms of convenience.

\subsection{Analyzing Fuben-eki Examples}

We call examples that provide users with the benefits of inconvenience fuben-eki examples. 


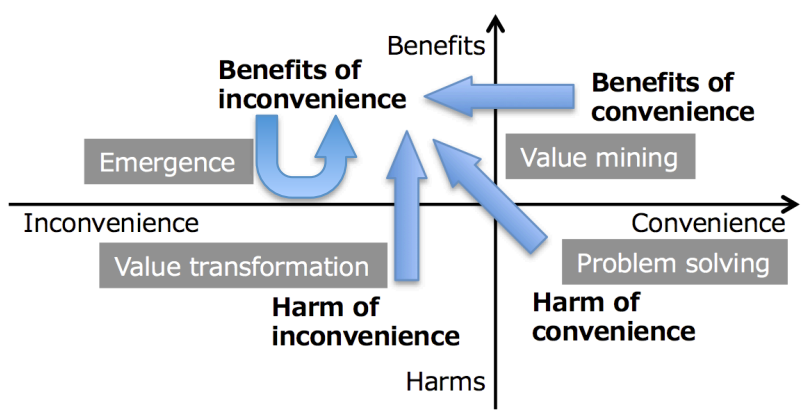

Fig. 2. Four types of ideation for fuben-eki systems

Not every inconvenient things have benefits. Somethings are just inconvenient and harmful. Such things are called negative examples and associated with points in the lower left quadrant of Fig. 2. Analyzing negative examples derives necessary conditions for approving fuben-eki systems:

- Benefits and inconvenience belong to the user.

- Benefits are directly derived from the inconveniences.

The former condition means that "your inconvenience is my benefit" is the worst case. The latter condition requires that benefits of inconvenience are not derived without inconvenience.

Analyzing positive examples derives notions that which kind of inconvenience is in what sense beneficial. Those notions can be such support tools as reported in [2] for synthesizing new fuben-eki systems. Positive examples are associated with points in the upper left quadrant of Fig. 2.

\section{Synthesizing Fuben-eki Systems}

. Conventional ideation support methods basically consist of divergence and convergence. At the divergence thinking stage, the quantity of ideas is required rather than their qualities, and criticism is forbidden. They are known as rules of Brainstorming. In this case, inconvenience is passively accepted. Namely, even the ideas are inconvenient, they are not eliminated.

On the other hand, this section positively utilizes inconvenience as a key to ideations. Many design workshops and design practices for designing fubeneki systems have been conducted. In the workshop, participants are required to design systems focusing on making systems inconvenient. It is not only a requirement but also an activator of ideation.

Observing such activities revealed that ideation processes can be classified into three types that can also correspond to three types of transition of the quadrants as depicted by arrows shown in Fig. 2. 


\subsection{Ideation through Problem Solving}

The normal way of engineering is based on problem-solving. Roughly, the process consists of two steps: formulating a problem, and solving it by developing a new system. This process can be applied to developing fuben-eki systems as well, i.e., formulating harm of convenience as a problem and solving it by making target systems inconvenient. This process can be called problem-solving based ideation.

The arrow in Fig. 2 from the right lower quadrant to the left upper quadrant illustrates this style of ideation. Namely, it is the transition of harms of convenience to benefits of inconvenience.

Degradation of navigation system: Degrade navigation system is one example designed by problem-solving based ideation. Navigation systems are generally convenient because they show clear and accurate information of maps. Unfortunately, the convenience deflates our motivation to remember the environment we are walking through. It is known as a problem called Cognitive Disuse Atrophy [3].

By using a navigation system, we only need to follow the fragments of direction to reach our destination, without exploring our surroundings or proactively understanding the area we are moving through. Therefore, we do not remember the roads. Using a navigation system reduce pleasurable explorations to merely idle transportation.

Degrade navigation system was developed as a solution to this problem. The normal navigation system was improved by introducing a novel inconvenience: map degradation [4]. This new system erases gradually the trails followed by users gradually from the map shown on the display of the navigation system. Compared with the normal navigation system, degrade navigation system is inconvenient, because users need to recall the surroundings of the trails when they use the system again; the system encourages users to remember landmarks more precisely. Fig. 3 shows the interface of Degradation Navi.

Barrie Aree: Barrier-Aree (Aree is a Japanese word that means existence) is another example of problem-solving based ideation. Compared with barrierfree, barrier-aree is an inconvenient design principle of residence because such minor obstacles as differences in floor levels are introduced to the living space on purpose. Residents are required their time and effort to walk through living spaces but barriers maintain physical and cognitive abilities of residents.

Barrier-aree was designed for solving the problem of physical and cognitive Disuse Atrophy [3] of residents of barrier-free residences.

\subsection{Ideation via Value Mining}

The second type of ideation methods is called value-mining. The major difference between problem-solving and value-mining is the supposition of problems to be solved. The Value-mining method does not expect problems of the target system. 


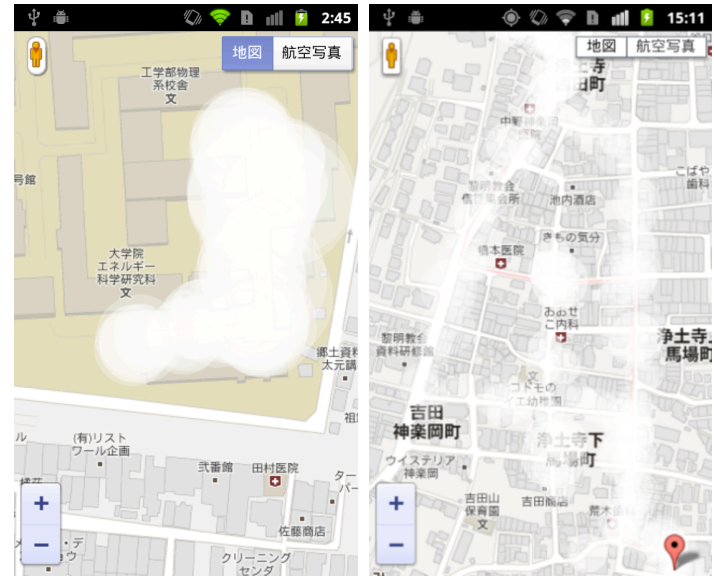

Fig. 3. Screenshot of Degradation Navi interface

The upper right quadrant in Fig. 2 represents happy states where the target system is convenient and users feel that it is beneficial. That is, there are no problems.

The Value-mining method starts from this happy state of the target system. At first, the target system is re-designed to be inconvenient one that requires more time and effort for using the system. Then, latent benefits are occasionally mined from the newly designed systems.

Conceptually, such ideation is a shift from the upper right to the upper left quadrant in Fig. 2. Namely, it is the transition of benefits of convenience to benefits of inconvenience.

Prime number ruler: Figure 4 shows an example of the results of such ideation: a prime number ruler, which is an original item of Kyoto University. A conventional ruler is only a convenient tool for measuring length, but an inconvenient prime number ruler allows users to devise their own ways to measure length.

At first, a ruler is selected as the target system to re-design. The normal ruler has no problem but participants of the design workshop are required to make rulers inconvenient. One of the ways to force users of rulers more consumption of time and efforts is restricting the tick marks to just prime numbers. The second step is mining benefits from prime number ruler. One benefit is allowing users to make a positive contribution to their tasks. A simple subtraction is required for measuring the length of a non-prime number. As a result, the task of measuring length requires users positive contribution.

Shared Space: Shared space can be seen as a political scheme of traffic designed by applying the value-mining method. It is not just a traffic scheme, but a design philosophy for creating good residential streets. A shared space design removes 


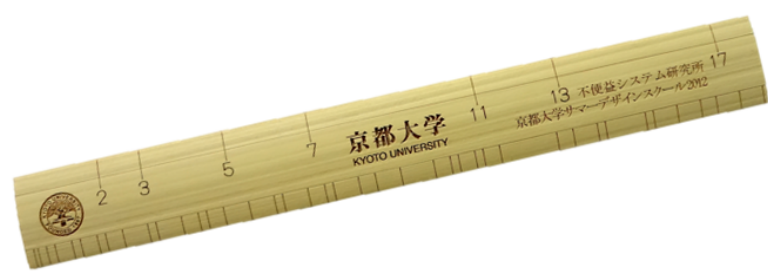

Fig. 4. Prime number ruler

such traffic controls as traffic lights, excessive signage, and road markings. The easiest way to control traffic is separation. Separating the roads for automobiles, bicycles, and foot passenger seems to bring safety of the space. On the other hand, shared space design enforce automobiles, bicycles, and foot passenger to share space.

It is inconvenient because the subject of keeping safety is not public administrations but people who exist in the space. This inconvenience leads the "sense of agency" to the people and fosters civility which enables users of the space to move safely through the space using social cues.

\subsection{Ideation by Emergence}

The third type of ideation is based on the emergent design that is different from both problem-solving and value-mining types. This type of ideation requires designers to shove many fuben-eki designs into their brain and to extract their essences without representing them by linguistic concepts. Then this method generates ideas what pops into the mind of designers.

Conceptually, such ideation is a shift within the upper left quadrant in Fig. 2. Namely, it is the transition of some benefits of inconvenience to a new benefit of inconvenience.

This type of ideation is the most non-systematic one, and practitioners of this ideation method are expected expert skills of ideation. Professional designers call this method one hundred knocks. This style of thought requires accumulated knowledge and the ability to change knowledge flexibly. Analogical thinking or analogical transfer are frequently used in this type of ideation.

Note that the emergent idea may have the opposite which has harms of convenience. The opposite can be the start point of problem-solving type of ideation, but if the process of ideation is based on emergent, the emerged idea is the production of this third type of ideation.

Gesture Unlock System: One of the examples of the production of emergentbased ideation is gesture unlock of smartphone. Considering a method for unlocking a smartphone as the theme of design workshop, we conducted a battle-style 
brain-storming [5] and got a hundred ideas. Among them, a gesture-unlocking mechanism was proposed.

To unlock her smartphone, the user of this unlocking system has to shake the phone with almost the same movement as she registered in advance. It is an inconvenient system because unlocking is almost impossible when an arbitrary gesture is registered. Muscle memory is required to register gestures.

That is, this system provides users with a subjective benefit called personalization [6]. Muscle memory depends on the body of each person, and the gesture cannot be replicated by others.

Talking-Ally: Talking-Ally [7] can be seen as a result of emergent-based ideation. It is an utterance generation system for communication robots with disfluencies. Generally, convenient communication aims clear, correct, and fluent utterance. From this point of view, disfluency is inconvenient property. On the other hand, the speeches generated by Talking-Ally are not fluent. Based on the assumption that disfluencies were created as a result of the interactions between the speaker and the hearer, Talking-Ally is developed.

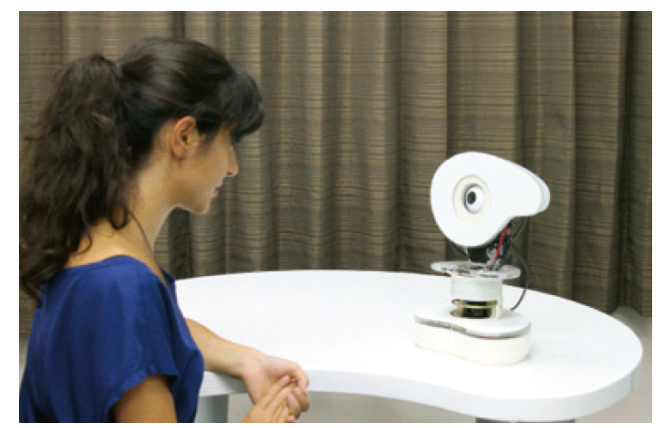

Fig. 5. Talking Ally is a utterance generation system for communication robot with disfluencies [7]

\section{Tools for Supporting Synthesize Fuben-eki Systems}

\subsection{Brainstorming Battle}

Brainstorming battle [5] is known as an effective tool for supporting ideation of fuben-eki systems. Brainstorming is one of the most famous divergent thinking methods, where participants have to keep four rules; criticism forbidden, freewheeling encouraged, quantity, and combine and expand. The method has such problems as "participants do not follow the rules," "some participants do not provide any idea," while it is useful for an idea generation. Brainstorming battle is a reformed scheme of brainstorming where participants are divided into some 
teams and all of them compete for an idea with others in the battle style. It solves the problems of normal brainstorming.

Brainstorming battle is effective for enhancing ideation processes, especially for emergent-based ideation. Namely, it is a scheme for group-work to emerge a new idea of a fuben-eki system.

\subsection{Knowledge-base system and card-type tools for fuben-eki design}

To support designing fuben-eki systems, we developed a knowledge-base system [8] and a card-type tool [2]. As a result of analyzing almost 100 positive examples of fuben-eki design from the point of view of the relationship between inconvenience and benefit, inconvenience-oriented benefits are classified into eight categories [8]. The green cards (shown in Fig. 6) show these eight categories.

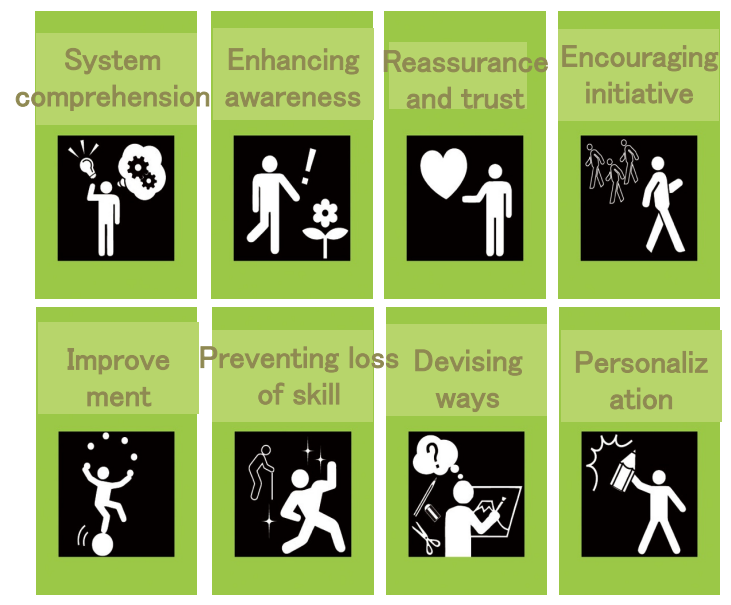

Fig. 6. Fuben-eki cards show example of benefits of inconvenience

The knowledge-base system is based on TRIZ, which is a theory for inventive problem-solving. The knowledge-base of original TRIZ is the result of analyzing millions of patent claims that provides about 40 principles for technical innovations. A tool for searching for appropriate principles called Contradiction Matrix is also provided.

We extracted 12 principles for designing fuben-eki systems by analyzing positive examples of fuben-eki design and created a tool called Fuben-eki Matrix. The principles are shown on the yellow cards as shown in Fig. 7. The matrix was implemented on a web application [8] and a card-type tool [2]

Yellow cards show the variation of the way to make systems inconvenient. Therefore, they are useful at the first step of the value-mining method where designers are asked to make the target system inconvenient. Green cards show 
8 categories of benefits that are derived from inconvenience. Therefore, they are useful at the second step of the value-mining method where designers are asked to mine benefits from the results of the first step.

The problem-solving method also utilizes cards. Green cards help designers to formulate harms of convenience at the first step of problem-solving, and yellow cards help to find the way to make the target system inconvenient for resolving the harms. The web system of Fuben-eki Matrix was designed for supporting designers following the process of problem-solving methods.

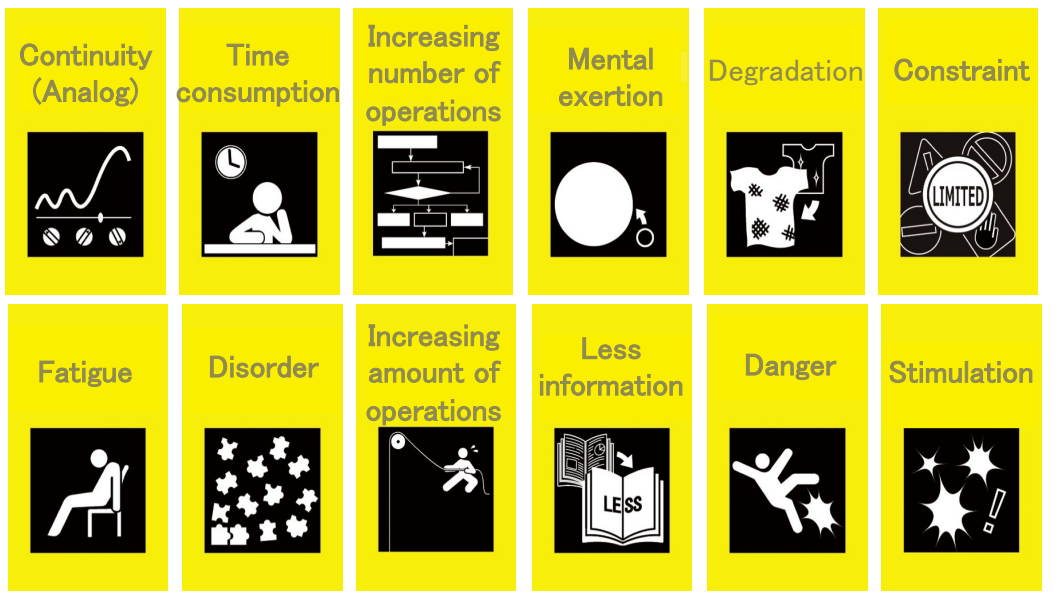

Fig. 7. Fuben-eki principle cards show ways to make system inconvenient

\subsection{Case-base system for fuben-eki design}

Fuben-eki matrix and Fuben-eki cards are based on principles that are derived from the abstractions of the examples. In contrast, we developed a case-base system [9] that does not rely on abstract principle but on analogies to examples.

In the case-base, positive examples of fuben-eki design are registered based on the following premises:

factors of inconvenience: fuben-eki must originate from inconvenience, main task: fuben-eki need to be collateral effects of achieving main tasks, comparison examples: convenience/inconvenience are comparative.

The positive examples are represented by at least their main tasks, their comparison examples, and the factors that are inconvenient. Embracing these notions, each example is represented by the following three types:

common type $(\chi)$ : common attributes of the fuben-eki examples and its comparison example, 
specific type $(\sigma)$ : objective functions specific to the fuben-eki example, fuben-eki type $(\phi)$ : subjective functions felt by users who use the fuben-eki example.

The process to use the case-base system is as follows:

1. select common type $(\chi)$ that are related to the target system $(t)$ from the list of $\chi$.

2. select desirable fuben-eki type $(\phi)$ from the list of $\phi$.

3. select specific type $(\sigma)$ that are related to $t$ and selected $\phi$ from the list of $\sigma$.

Through this process, the system generates appropriate lists of $\phi$ and $\sigma$, then generates useful reference for positive examples and their attribute for ideating new design.

This process supports the analogy between positive examples and the target system. Therefore, the case-base system effectively supports the ideation of the emergent-based method.

\section{Conclusions}

This paper discussed benefits of inconvenience as a new direction of HMI design. It is an alternative direction of uncritical pursuits to convenience. Of course, this direction does not insist on the uncritical pursuit of inconvenience. There exist harmful inconvenience as well as beneficial inconvenience. We need to eliminate the harmful one and to focus on the beneficial one.

This paper showed three types of methods to ideate systems that incorporate the benefits of inconvenience. The types are based on problem-solving, value-mining, and emergence. The start points of three types of thoughts are associated with the three of quadrants that is defined by orthogonal axises of convenience/inconvenience and benefits/harms.

There remains another quadrant that reflects harms of inconvenience. It must be happy to change from the state of harms of inconvenience to the benefits of inconvenience. Some design examples are known as implementations of this state change. A hotel is inconvenient because it is hard to access, but the inconvenience gives us a scarcity premium. A restaurant is inconvenient because it is hidden from main streets, but the inconvenience provides us with a sense of a safe house.

Another design of this fourth type was given by Hiraoka [10]. Based on psychological theories with respect to motivation, an eco-driving system was designed that transform annoying labor into a worthy activity.

\section{References}

1. Kawakami, H.: Toward systems design based on benefit of inconvenience. J. of Human Interface. 11(1), 125-134 (2009) (in Japanese)

2. Hasebe, Y., Kawakami, H, Hiraoka, T., and Naito, K.: Card-type tool to support divergent thinking for embodying benefits of inconvenience. Web Intelligence 13 (2015) 93-102, DOI 10.3233/WEB-150312, IOS Press 
3. Miwa, K., Terai, H.: Theoretical investigation on disuse atrophy resulting from computer support for cognitive tasks. Engineering Psychology and Cognitive Ergonomics (LNCS, volume 8532), pp 244-254, Springer (2014)

4. Kitagawa, H., et al.: Degrading navigation system as an explanatory example of "benefits of inconvenience." Proc SICE Annaul Confirmed. 1738-1742 (2010)

5. Hiraoka, T., Hasebe, Y., Kawakami, H.: Brainstorming battle. Proc. of JSAI15, https://doi.org/10.11517/pjsai.JSAI2015.0_1D40S22a5 (2015)

6. Norman, D. A.: Emotional design. New York: Basic Books (2004).

7. Ohshima, N., Ohyama, Y., Odahara, Y., Silva, P, Okada, M.: Talking-Ally: The influence of robot utterance generation mechanism on hearer behaviors. Int. J. of Social Robotics, 7(1), pp.51-62 (2015).

8. Naito, K., Kawakami, H., Hiraoka, T.: Design support method for implementing benefit of inconvenience inspired by TRIZ. 12th ETRIZ TRIZ future conference, 351-356 (2012)

9. Kawakami, H., Hiraoka, T., Riku, S.: Ideation support based on infomorphsim for designing beneficial inconvenience. Intelligent and Evolutionary Systems, Proc. in Adaptation, Learning and Optimization, vol 5. Springer, 255-266 (2015)

10. Hiraoka, T., et al.: Eco-driving support system to encourage spontaneous fuelefficient driving behavior. J. of the Society of Instrument and Control Engineers. 48(11), 754-763 (2012) 